ered a dramatic finding-a respiratory syncytial virus (RSV) infection appeared to trigger the onset of type 2 diabetes, to which Snyder was already genetically predisposed (Cell 148, 1293-1307, 2012). "It's not hard science, but anecdotally there seems to be something to it," Snyder says. Intervention, in the form of a diet and exercise plan coupled with lowdose aspirin, helped to lower his blood sugar and glycosylated hemoglobin levels. Snyder has since scaled up the iPOP project to 100 subjects-including a woman with a BRCA1 mutation but who had no family history of $B R C A$-associated cancers-and has plans to recruit more, all of whom will undergo WGS as well profiling of their respective transcriptomes, DNA methylomes, proteomes, autoantibody repertoires and gut microbiomes. The total cost is about $\$ 50,000-\$ 100,000$ per participant. Although WGS may cost less than $\$ 2,000$, a detailed interpretation can push the cost as high as $\$ 20,000$, he says. The depth of the analysis is for discovery purposes-a lighter touch would be used for more routine analysis of larger populations, Snyder says.

Whether these approaches are an efficient way of uncovering clinically useful information remains an open question for now. Eleftherios Diamandis, head of clinical biochemistry at the University of Toronto and at Mount Sinai Hospital in Toronto, is the most vocal critic of Hood's endeavor (BMC Med. 13, 5, 2015). He characterizes the effort as screening rather than a longitudinal study. "Population screening involves the testing of asymptomatic individuals in the hope you will find some occult disease," he says. It is likely, he says, to lead to overtesting and overdiagnosis. Detection of indolent disease could lead to risky

"What I find very
disconcerting is the
medical establishment has
no problem using family
histories in managing
people's health"

A second category of firms, which is rolling out low-cost, basic analytical services to very large populations, has emerged in parallel with those focusing on in-depth, tailored analysis. 23andMe, of Mountain View, California, genotyped its millionth customer in June and claims that $80 \%$ of its customers have allowed their data to be used in its in-house genetic research. Theranos, of Palo Alto, California, aims to re-engineer blood testing at both a technological level and a business level. The privately held company, which has a reported valuation of $\$ 9$ billion, has developed new methods for collecting and analyzing low-volume samples, drawn via a pinprick, and it is commercializing its offerings through a network of retail pharmacy chains. An online team of licensed primary care physicians helps patients to interpret their results.

Linda Avey, a co-founder of 23andMe, has re-emerged in a new startup, Woodside, California-based We Are Curious, which allows users to track and chart their phenotypic and activity data using a 'wellness ring'. How these 'light-touch' efforts will stack up against the more comprehensive investigations offered by Arivale and others remains an open question. "I expect there will exist both individual, targeted approaches and more aggregate data approaches, depending on the indication, utility and evidence," says Daphne Zohar, co-founder and CEO of Boston-based healthcare innovation and investment firm PureTech.

How they will influence medical practice also remains to be seen. Although these emerging ventures are quick to embrace the 'disruptive' tag, none can actually supplant medical practice. or harmful interventions that do not benefit the patient, and false positives could cause unnecessary anxiety. What's more, most genetic variation is still poorly understood. "I know this is going to be cleared up as people characterize the variants, but it's going to take at least ten years," he says.

Snyder counters that existing medical routines are far from optimal. "What I find very disconcerting is the medical establishment has no problem using family histories in managing people's health," he says. "Genomics is probably better than family histories in many respects." Nor is there any evidence, he says, that biological analysis has aroused anxiety in participants.
Once the platforms and data mature, they should add more scientific rigor to clinical decision-making, but they could influence the evolution of health and medicine more profoundly. The discovery of new interventions is ongoing - that work has barely started, in fact. "Probably we have $10 \%$ to $20 \%$ of the actionable possibilities that are out there," says Hood. From a competitive standpoint, it's still all to play for. Although rapid technological development and falling costs have made it easier than ever to generate biological data, finding useful ways of interpreting and applying them will be the greater challenge.

Cormac Sheridan Dublin

\section{UK biotech smashes financing record}

Immunocore of Abingdon, UK, secured a record $\$ 320$ million in a series $A$ round in July. It is Europe's largest-ever venture round and the second biggest worldwide, behind Moderna Therapeutics' $\$ 450$ million raised in January (Nat. Biotechnol. 33, 199, 2015). New investors Woodford Investment Management, Malin Crop and Eli Lilly participated alongside undisclosed existing investors. Immunocore said it will use the funding to accelerate its pipeline of therapeutics called ImmTACS. These are a new class of bispecific molecules that combine a monoclonal T-cell receptor fused to an anti-CD3 antibody fragment that recruits T cells to the tumor. Immunocore's lead candidate IMCgp100 is in phase 2a testing for late-stage melanoma. The company has already signed partnerships with Lilly, Genentech, GlaxoSmithKline and MedImmune.

\section{BEAM to fight antimicrobial resistance}

A group of European biotech companies with novel approaches to tackle antimicrobial resistance formed the BEAM (Biotechs from Europe innovating in Antimicrobial Resistance) Alliance earlier this year. The 39 small to medium-sized enterprises involved in developing antibiotic drugs or infectious disease diagnostics in one way or another, from ten countries across the continent, joined forces with a common interest in using innovation to prevent resistance from arising in both old and newer antimicrobial products. The BEAM Alliance and its mission to improve investment and commercial viability for new products that combat antimicrobial resistance tracks closely with several other prominent groups drawing attention to this global public health issue. For instance, in June, the heads of state who were gathered at the G7 countries meeting in Germany said in the Leaders' Declaration that they fully support the World Health Organization's Global Action Plan on Antimicrobial Resistance. They further said that they will foster prudent use of antibiotics and help to stimulate basic research, research on epidemiology, infection prevention and control, and the development of new antibiotics, alternative therapies, vaccines and rapid point-of-care diagnostics.

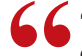

"Biomedical research will always be closer to Sisyphus than a runaway trainand the last thing we need is a lobby of socalled ethicists helping to push the rock down the hill." Steven Pinker, Harvard psychologist, argues in an op-ed piece, advising bioethicists to "get out of the way" of biomedical progress. (Boston Globe, 1 August 2015) 